\title{
Summary
}

\section{RECOMMENDATIONS FOR ROTAVIRUS IMMUNIZATION PROGRAMS}

\author{
Canadian Immunization Committee*
}

\section{BACKGROUND}

Rotavirus is a common cause of gastroenteritis in children and can vary from asymptomatic to severe disease. Based on scientific evidence, the National Advisory Committee on Immunization (NACl) recommends rotavirus vaccine in infants for the prevention of this common viral gastroenteritis. The Canadian Immunization Committee (CIC) considered the programmatic issues of this vaccine and its appropriateness for inclusion in provincial and territorial routine immunization schedules. This document is a brief summary of the full CIC rotavirus vaccine statement. (1)

\section{APPROACH}

A Task Group of the CIC considered the burden of illness, vaccine safety and efficacy, cost-effectiveness, acceptability and feasibility, and ethical and legal considerations. It then made recommendations on a universal rotavirus vaccine program, an evaluation strategy and future research questions.

\section{FINDINGS}

Rotavirus is a common cause of gastroenteritis in children and can have serious sequelae, especially for those less than 2 years of age. In Canada, 1 in 7 children with rotavirus will seek health care, 1 in 20 will visit an emergency department or be hospitalized, and 1 in 62 will be hospitalized. Two oral rotavirus vaccines approved for use in Canada, RotaTeq ${ }^{\circledR}$ and Rotarix ${ }^{\mathrm{TM}}$, are both effective and safe against the common serotypes. Intussusception rarely occurs following immunization, and may also occur with infection. A careful safety evaluation has concluded that the benefits of rotavirus vaccine outweigh this risk. A systematic review of costeffectiveness studies suggested that these vaccines are cost-effective even when herd immunity is not considered. Rotarix ${ }^{\mathrm{TM}}$ was found to be more cost-effective than RotaTeq ${ }^{\circledR}$ in the Canadian studies. Acceptability studies have found that parents like the fact that rotavirus vaccine is oral and effective at preventing severe disease and would rely on physician recommendation regarding its use. Rotavirus vaccination is feasible as it fits well into the current routine immunization schedule.

\section{RECOMMENDATIONS}

The CIC supports the routine use of rotavirus vaccines in infants without contraindications based on the recommendations of $\mathrm{NACl}$, Canadian studies demonstrating its cost-effectiveness and Canadian studies indicating a high degree of acceptability and feasibility. An evaluation framework for rotavirus immunization programs is recommended to:

1. monitor the burden of disease in Canada;

2. assess severity of infections;

3. track vaccine coverage in the target population; and

4. monitor vaccine safety. 
Additional identified areas of research include uptake studies, assessing herd immunity and the use of rotavirus vaccine in premature infants.

\section{REFERENCE}

1. Canadian Immunization Committee (CIC). (2013). Recommendations for Rotavirus Immunization Programs.

* The Canadian Immunization Committee (CIC) provides operational and technical advice related to immunization policies and programs in Canada. CIC consists of representatives from provincial and territorial jurisdictions, the National Advisory Committee on Immunization (NACl), the American Advisory Committee on Immunization Practices (ACIP) and Health Canada's Biologics and Genetics Therapies Directorate (BGTD), Marketed Health Products Directorate (MHPD), and First Nations and Inuit Health Branch. 